\title{
Subclasses of p-valent meromorphic functions involving certain operator
}

\author{
Adela O. Mostafa and Mohamed K. Aouf
}

\begin{abstract}
In this paper we investigate some inclusion relationships of two new subclassses of meromorphically p-valent functions, defined by means of a linear operator. We also study some integral preserving properties and convolution properties of these classes.
\end{abstract}

Mathematics Subject Classification (2010): 30C45.

Keywords: Analytic, p-valent, meromorphic, linear operator, differential subordination, inclusion relationships.

\section{Introduction}

Let $\sum_{p}$ denote the class of all meromorphic functions $f$ defined by:

$$
f(z)=z^{-p}+\sum_{k=1}^{\infty} a_{k-p} z^{k-p} \quad(p \in \mathbb{N}=\{1,2, \ldots\}),
$$

which are analytic and $p$-valent in a punctured unit disk $\mathbb{U}^{*}=\{z: z \in \mathbb{C}$ and $0<|z|<1\}=\mathbb{U} \backslash\{0\}$.

The class of analytic functions of the form

$$
f(z)=z+\sum_{k=2}^{\infty} a_{k} z^{k}, z \in \mathbb{U}
$$

is denoted by $\mathbb{A}$. The functions of this class is called starlike of order $\gamma, 0 \leq \gamma<1$ if

$$
\Re \frac{z f^{\prime}(z)}{f(z)}>\gamma
$$

and called prestarlike of order $\gamma, \gamma<1$ if

$$
\frac{z}{(1-z)^{2(1-\gamma)}} * f(z) \in S^{*}(\gamma)
$$

we denote by $S^{*}(\gamma)$ and $R(\gamma)$ the classes of stalike and prestarlike of order $\gamma$. 
If $f$ and $g$ are analytic functions in $\mathbb{U}$, we say that $f$ is subordinate to $g$, written $f \prec g$ if there exists a Schwarz function $w$, which (by definition) is analytic in $\mathbb{U}$ with $w(0)=0$ and $|w(z)|<1$ for all $z \in \mathbb{U}$, such that $f(z)=g(w(z)), z \in \mathbb{U}$. Furthermore, if the function $g$ is univalent in $\mathbb{U}$, then we have the following equivalence (see $[2,5,6]$ ):

$$
f(z) \prec g(z) \Leftrightarrow f(0)=g(0) \text { and } f(\mathbb{U}) \subset g(\mathbb{U}) .
$$

For functions $f(z) \in \sum_{p}$ given by (1.1) and $g(z) \in \sum_{p}$ given by

$$
g(z)=z^{-p}+\sum_{k=1}^{\infty} b_{k-p} z^{k-p}
$$

the Hadamard product of $f(z)$ and $g(z)$ is given by:

$$
(f * g)(z)=z^{-p}+\sum_{k=1}^{\infty} a_{k-p} b_{k-p} z^{k-p}=(g * f)(z) .
$$

Using the operator $Q_{\beta, p}^{\alpha}: \sum_{p} \rightarrow \sum_{p}$ defined by (see [1]):

$$
Q_{\beta, p}^{\alpha} f(z)=\left\{\begin{array}{cc}
z^{-p}+\frac{\Gamma(\alpha+\beta)}{\Gamma(\beta)} \sum_{k=1}^{\infty} \frac{\Gamma(k+\beta)}{\Gamma(k+\beta+\alpha)} a_{k-p} z^{k-p} & (\alpha>0 ; \beta>-1) \\
f(z) & (\alpha=0 ; \beta>-1) .
\end{array}\right.
$$

Mostafa [8] defined the operator $H_{p, \beta, \mu}^{\alpha}: \Sigma_{p} \rightarrow \Sigma_{p}$ as follows:

First put

$$
G_{\beta, p}^{\alpha}(z)=z^{-p}+\frac{\Gamma(\alpha+\beta)}{\Gamma(\beta)} \sum_{k=1}^{\infty} \frac{\Gamma(k+\beta)}{\Gamma(k+\beta+\alpha)} z^{k-p} \quad(p \in \mathbb{N})
$$

and let $G_{\beta, p, \mu}^{\alpha *}$ be defined by

$$
G_{\beta, p}^{\alpha}(z) * G_{\beta, p, \mu}^{\alpha *}(z)=\frac{1}{z^{p}(1-z)^{\mu}}(\mu>0 ; p \in \mathbb{N}) .
$$

Then

$$
H_{p, \beta, \mu}^{\alpha} f(z)=G_{\beta, p}^{\alpha *}(z) * f(z) \quad\left(f \in \Sigma_{p}\right) .
$$

Using (1.3)-(1.5), we have

$$
H_{p, \beta, \mu}^{\alpha} f(z)=z^{-p}+\frac{\Gamma(\beta)}{\Gamma(\alpha+\beta)} \sum_{k=1}^{\infty} \frac{\Gamma(k+\beta+\alpha)(\mu)_{k}}{\Gamma(k+\beta)(1)_{k}} a_{k-p} z^{k-p},
$$

where $f \in \Sigma_{p}$ is in the form (1.1) and $(\nu)_{n}$ denotes the Pochhammer symbol given by

$$
(\nu)_{n}=\frac{\Gamma(\nu+n)}{\Gamma(\nu)}= \begin{cases}1 & (n=0) \\ \nu(\nu+1) \ldots(\nu+n-1) & (n \in \mathbb{N}) .\end{cases}
$$

It is readily verified from (1.6) that ( see [8])

$$
z\left(H_{p, \beta, \mu}^{\alpha} f(z)\right)^{\prime}=(\alpha+\beta) H_{p, \beta, \mu}^{\alpha+1} f(z)-(\alpha+\beta+p) H_{p, \beta, \mu}^{\alpha} f(z)
$$

and

$$
z\left(H_{p, \beta, \mu}^{\alpha} f(z)\right)^{\prime}=\mu H_{p, \beta, \mu+1}^{\alpha} f(z)-(\mu+p) H_{p, \beta, \mu}^{\alpha} f(z) .
$$


It is noticed that, putting $\mu=1$ in (1.6), we obtain the operator

$$
H_{p, \beta, 1}^{\alpha} f(z)=H_{p, \beta}^{\alpha} f(z)=z^{-p}+\frac{\Gamma(\beta)}{\Gamma(\alpha+\beta)} \sum_{k=1}^{\infty} \frac{\Gamma(k+\alpha+\beta)}{\Gamma(k+\beta)} a_{k-p} z^{k-p} .
$$

Let $\mathbb{P}$ be the class of functions $h(z)$ with $h(0)=1, \operatorname{Re} h(z)>0$ which are convex univalent in $\mathbb{U}$.

For $p, n \in \mathbb{N}, \in_{n}=e^{2 \pi / n}$, let

$$
f_{n}^{\mu}(\alpha)(z)=\frac{1}{n} \sum_{j=0}^{n-1} \varepsilon_{n}^{j p} H_{p, \beta, \mu}^{\alpha} f\left(\varepsilon_{n}^{j} z\right)=z^{-p}+\ldots, f \in \sum_{p} .
$$

By (1.7) and (1.8), $f_{n}^{\mu}(\alpha)(z)$ satisfies:

$$
z\left(f_{n}^{\mu}(\alpha)(z)\right)^{\prime}=(\alpha+\beta) f_{n}^{\mu}(\alpha+1)(z)-(\alpha+\beta+p) f_{n}^{\mu}(\alpha)(z)
$$

and

$$
z\left(f_{n}^{\mu}(\alpha)(z)\right)^{\prime}=\mu f_{n}^{\mu+1}(\alpha)(z)-(\mu+p) f_{n}^{\mu}(\alpha)(z) .
$$

Definition 1.1. For $h \in \mathbb{P}, f \in \sum_{p}, f_{n}^{\mu}(\alpha)(z) \neq 0, z \in \mathbb{U}^{*}, S_{n}^{\mu}(\alpha, h)$ is the class of functions $f$ satisfying:

$$
-\frac{z\left(H_{p, \beta, \mu}^{\alpha} f(z)\right)^{\prime}}{p f_{n}^{\mu}(\alpha)(z)} \prec h(z)
$$

and $K_{n}^{\mu}(\alpha, h)$ is the class of functions $f$ satisfying:

$$
-\frac{z\left(H_{p, \beta, \mu}^{\alpha} f(z)\right)^{\prime}}{p g_{n}^{\mu}(\alpha)(z)} \prec h(z),
$$

where $g_{n}^{\mu}(\alpha)(z) \neq 0$, is defined as in $(1.10)$.

To prove our results, we need the following Lemmas.

Lemma 1.2. [3] Let $\beta, \gamma \in \mathbb{C}, \beta \neq 0, h$ be convex univalent with $\Re\{\beta h(z)+\gamma\}>0$ and $q$ be an analytic function such that $q(0)=h(0)$. If

$$
q(z)+\frac{z q^{\prime}(z)}{\beta q(z)+\gamma} \prec h(z)
$$

then

$$
q(z) \prec h(z) .
$$

Lemma 1.3. [7] Let $h$ be convex univalent and $w$ be analytic, $\Re w \geq 0$. If the analytic function $q$ satisfies $q(0)=h(0)$ and

$$
q(z)+w(z) z q^{\prime}(z) \prec h(z)
$$

then $q(z) \prec h(z)$.

Lemma 1.4. [9] For $\alpha<1, f \in R(\alpha)$ and $\varphi \in S^{*}(\alpha)$, we have for any analytic function $F$ in $\mathbb{U}$,

$$
\frac{f *(\varphi F)}{f * \varphi}(\mathbb{U}) \subset \overline{c o}(F(\mathbb{U})
$$

where $\overline{c o}(F(\mathbb{U})$ is the convex hull of $(F(\mathbb{U})$. 


\section{Main results}

Theorem 2.1. If $f \in S_{n}^{\mu}(\alpha, h)$, then

$$
-\frac{z\left(f_{n}^{\mu}(\alpha)(z)\right)^{\prime}}{p f_{n}^{\mu}(\alpha)(z)} \prec h(z),
$$

where $f_{n}^{\mu}(\alpha)(z)$ is defined as in (1.10).

Proof. From (1.10), we have:

$$
\begin{aligned}
f_{n}^{\mu}(\alpha)\left(\in_{n}^{j} z\right) & =\frac{1}{n} \sum_{t=0}^{n-1} \varepsilon_{n}^{j t} H_{p, \beta, \mu}^{\alpha} f\left(\varepsilon_{n}^{j+t} z\right) \\
& =\frac{\varepsilon_{n}^{-j p}}{n} \sum_{t=0}^{k-1} \varepsilon_{n}^{(j+t) p} H_{p, \beta, \mu}^{\alpha} f\left(\varepsilon_{n}^{j+t} z\right) \\
& =\varepsilon_{n}^{-j p} f_{n}^{\mu}(\alpha)(z)
\end{aligned}
$$

and

$$
\left(f_{n}^{\mu}(\alpha)(z)\right)^{\prime}=\frac{1}{n} \sum_{j=0}^{n-1} \varepsilon_{n}^{j(p+1)}\left(H_{p, \beta, \mu}^{\alpha} f\left(\varepsilon_{n}^{j+t} z\right)\right)^{\prime} .
$$

By (2.2) and (2.3), we have

$$
\begin{aligned}
-\frac{z\left(f_{n}^{\mu}(\alpha)(z)\right)^{\prime}}{p f_{n}^{\mu}(\alpha)(z)} & =-\frac{1}{n} \sum_{j=0}^{n-1} \frac{\varepsilon_{n}^{j(p+1)}\left(H_{p, \beta, \mu}^{\alpha} f\left(\varepsilon_{k}^{j} z\right)\right)^{\prime}}{p f_{n}^{\mu}(\alpha)(z)} \\
& =-\frac{1}{n} \sum_{j=0}^{n-1} \frac{\varepsilon_{n}^{j}\left(H_{p, \beta, \mu}^{\alpha} f\left(\varepsilon_{n}^{j} z\right)\right)^{\prime}}{p f_{n}^{\mu}(\alpha)(z)} .
\end{aligned}
$$

Since $f \in S_{n}^{\mu}(\alpha, h)$, we have,

$$
-\frac{\varepsilon_{n}^{j}\left(H_{p, \beta, \mu}^{\alpha} f\left(\varepsilon_{n}^{j} z\right)\right)^{\prime}}{p f_{n}^{\mu}(\alpha)(z)} \prec h(z),
$$

which leads to (2.1).

Theorem 2.2. For $\alpha+\beta>0, h \in \mathbb{P}$ with $\Re\{\alpha+\beta+p-p h(z)\}>0$ and for $f \in$ $S_{n}^{\mu}(\alpha+1, h), g_{n}^{\mu}(\alpha) \neq 0$, we have, $f \in S_{n}^{\mu}(\alpha, h)$.

Proof. Since $f \in S_{n}^{\mu}(\alpha+1, h)$, then the function

$$
q(z)=-\frac{\left.z\left(H_{p, \beta, \mu}^{\alpha} f(z)\right)\right)^{\prime}}{p f_{n}^{\mu}(\alpha)(z)},
$$

is analytic and $q(0)=1$. Applying (1.8) in (2.5), we have

$$
q(z) f_{n}^{\mu}\left(\alpha_{1}\right)(z)=-\frac{1}{p}\left[(\alpha+\beta) H_{p, \beta, \mu}^{\alpha+1} f(z)-(\alpha+\beta+p) H_{p, \beta, \mu}^{\alpha} f(z)\right] .
$$


Differentiating (2.6) and using (1.8) again, we have

$$
\left(\alpha+\beta+p+\frac{z\left(f_{n}^{\mu}(\alpha)(z)\right)^{\prime}}{f_{n}^{\mu}(\alpha)(z)}\right) q(z)+z q^{\prime}(z)=-\frac{(\alpha+\beta) z\left(H_{p, \beta, \mu}^{\alpha+1} f(z)\right)^{\prime}}{p f_{n}^{\mu}(\alpha)(z)} .
$$

Taking

$$
\phi(z)=-\frac{z\left(f_{n}^{\mu}(\alpha)(z)\right)^{\prime}}{p f_{n}^{\mu}(\alpha)(z)}
$$

we see that $\phi(z)$ is analytic, $\phi(0)=1$ and $(2.7)$ can be written as

$$
(\alpha+\beta+p-p \phi(z)) q(z)+z q^{\prime}(z)=-\frac{(\alpha+\beta) z\left(H_{p, \beta, \mu}^{\alpha+1} f(z)\right)^{\prime}}{p f_{n}^{\mu}(\alpha)(z)},
$$

that is

$$
q(z)+\frac{z q^{\prime}(z)}{\alpha+\beta+p-p \phi(z)}=-\frac{z\left(H_{p, \beta, \mu}^{\alpha+1} f(z)\right)^{\prime}}{p f_{n}^{\mu}(\alpha+1)(z)} .
$$

Since $f \in S_{n}^{\mu}(\alpha+1, h),(2.10)$ implies

$$
q(z)+\frac{z q^{\prime}(z)}{\alpha+\beta+p-p \phi(z)} \prec h(z) .
$$

Combining (2.11) and (2.8), we have

$$
\alpha+\beta+p-p \phi(z)=\frac{(\alpha+\beta) f_{n}^{\mu}(\alpha+1)(z)}{p f_{n}^{\mu}(\alpha)(z)} .
$$

Differentiating (2.12), we get

$$
\phi(z)+\frac{z \phi^{\prime}(z)}{\alpha+\beta+p-p \phi(z)}=-\frac{z\left(f_{n}^{\mu}(\alpha+1)(z)\right)^{\prime}}{p f_{n}^{\mu}(\alpha+1)(z)} .
$$

By Theorem 2.1, we have

$$
-\frac{z\left(f_{n}^{\mu}(\alpha+1)(z)\right)^{\prime}}{p f_{n}^{\mu}(\alpha+1)(z)} \prec h(z)
$$

which yields

$$
\phi(z)+\frac{z \phi^{\prime}(z)}{\alpha+\beta+p-p \phi(z)} \prec h(z) .
$$

Since $\Re\{\alpha+\beta+p-p h(z)\}>0$, by Lemma 1.2, we have $\phi(z) \prec h(z)$, which implies $\Re\{\alpha+\beta+p-p \phi(z)\}>0$. Applying Lemma 1.3 and from $(2.10)$, we have $q(z) \prec h(z)$ that is $f \in S_{n}^{\mu}(\alpha, h)$.

Theorem 2.3. Let $\alpha+\beta>0, h \in \mathbb{P}$ with $\Re\{\alpha+\beta+p-p h(z)\}>0$ and $f \in K_{n}^{\mu}(\alpha+1, h)$ with $g \in S_{n}^{\mu}(\alpha+1, h)$. Then, $f \in K_{n}^{\mu}(\alpha, h)$ provided $g_{n}^{\mu}(\alpha)(z) \neq 0$.

Proof. By Theorem 2.2, $g \in S_{n}^{\mu}(\alpha+1, h) \Rightarrow g \in S_{n}^{\mu}(\alpha, h)$ and by Theorem 2.1, we have

$$
\psi(z)=-\frac{z\left(g_{n}^{\mu}(\alpha)(z)\right)^{\prime}}{p g_{n}^{\mu}(\alpha)(z)} \prec h(z) .
$$


Let

$$
q(z)=-\frac{z\left(H_{p, \beta, \mu}^{\alpha} f(z)\right)^{\prime}}{p g_{n}^{\mu}(\alpha)(z)}
$$

Then, from (1.8), we have

$$
q(z) g_{n}^{\mu}(\alpha)(z)=-\frac{1}{p}\left[(\alpha+\beta) H_{p, \beta, \mu}^{\alpha+1} f(z)-(\alpha+\beta+p) H_{p, \beta, \mu}^{\alpha} f(z)\right] .
$$

Differentiating (2.16), we have

$$
(\alpha+\beta+p-p \psi(z)) q(z)+z q^{\prime}(z)=-\frac{(\alpha+\beta) z\left(H_{p, \beta, \mu}^{\alpha+1} f(z)\right)^{\prime}}{p g_{n}^{\mu}(\alpha)(z)} .
$$

Applying (1.11) for $g,(2.17)$ is equivalent to

$$
q(z)+\frac{z q^{\prime}(z)}{\alpha_{1}+p-p \psi(z)}=-\frac{z\left(H_{p, \beta, \mu}^{\alpha+1} f(z)\right)^{\prime}}{p g_{n}^{\mu}(\alpha+1)(z)} .
$$

Since $f \in K_{n}^{\mu}(\alpha+1, h)$, the above equation leads to

$$
q(z)+\frac{z q^{\prime}(z)}{\alpha_{1}+p-p \psi(z)} \prec h(z) .
$$

We have $\Re\{\alpha+\beta+p-p \psi(z)\}>0$ because $\Re\{\alpha+\beta+p-p h(z)\}>0$. Applying Lemma 1.3, for (2.19), we have $q(z) \prec h(z)$. That is $f \in K_{n}^{\mu}(\alpha, h)$.

Theorem 2.4. Let $h \in \mathbb{P}, \Re\{\mu+p-p h(z)\}>0$ and $f \in S_{n}^{\mu+1}(\alpha, h)$ such that $f_{n}^{\mu+1}(\alpha)(z) \neq 0$. Then $f \in S_{n}^{\mu}(\alpha, h)$.

Proof. Let $f \in S_{n}^{\mu+1}(\alpha, h)$,

$$
q(z)=-\frac{z\left(H_{p, \beta, \mu}^{\alpha} f(z)\right)^{\prime}}{p f_{n}^{\mu}(\alpha)(z)} .
$$

Applying (1.9) in (2.20), we have

$$
q(z) f_{n}^{\mu}(\alpha)(z)=-\frac{\mu}{p}\left[H_{p, \beta, \mu+1}^{\alpha} f(z)+\left(\frac{\mu+p}{p}\right) H_{p, \beta, \mu}^{\alpha} f(z)\right] .
$$

Differentiating (2.21) and putting

$$
\Phi(z)=-\frac{z\left(f_{n}^{\mu}(\alpha)(z)\right)^{\prime}}{p f_{n}^{\mu}(\alpha)(z)}
$$

simple computations leads to

$$
[\mu+p-p \Phi(z)] q(z)+z q^{\prime}(z)=-\left(\frac{\mu}{p}\right) \frac{z\left(H_{p, \beta, \mu+1}^{\alpha} f(z)\right)^{\prime}}{p f_{n}^{\mu}(\alpha)(z)} .
$$

Using (1.12), we have

$$
\mu+p-p \Phi(z)=\frac{\mu f_{n}^{\mu+1}(\alpha)(z)}{f_{n}^{\mu}(\alpha)(z)}
$$


So, $(2.23)$, reduces to

$$
q(z)+\frac{z q^{\prime}(z)}{\mu+p-p \Phi(z)}=-\frac{z\left(H_{p, \beta, \mu+1}^{\alpha} f(z)\right)^{\prime}}{p f_{n}^{\mu+1}(\alpha)(z)} \prec h(z),
$$

where $f \in S_{n}^{\mu+1}(\alpha, h)$. Also differentating (2.24), we have

$$
\Phi(z)+\frac{z \Phi^{\prime}(z)}{\mu+p-p \Phi(z)}=-\frac{z\left(f_{n}^{\mu+1}(\alpha) f(z)\right)^{\prime}}{p f_{n}^{\mu+1}(\alpha)(z)} .
$$

By Theorem 2.1, we have

$$
-\frac{z\left(f_{n}^{\mu+1}(\alpha) f(z)\right)^{\prime}}{p f_{n}^{\mu+1}(\alpha)(z)} \prec h(z) .
$$

Combining (2.26), (2.27) and the condition $\Re\{\mu+p-p h(z)\}>0$, we have $\Phi(z) \prec h(z)$, which leads to $\Re\{\mu+p-p \Phi(z)\}>0$ and so applying Lemma 1.3 to (2.25). we have $q(z) \prec h(z)$ which completes the proof of Theorem 2.4 .

Theorem 2.5. Let $h \in \mathbb{P}$ with $\Re\{\mu+p-p h(z)\}>0$ and $f \in K_{n}^{\mu+1}(\alpha, h)$ with $g \in$ $S_{n}^{\mu+1}(\alpha, h)$. Then, $f \in K_{n}^{\mu}(\alpha, h)$ provided $g_{n}^{\mu}(\alpha)(z) \neq 0$.

Proof. By Theorem 2.4, $g \in S_{n}^{\mu+1}(\alpha, h) \Rightarrow g \in S_{n}^{\mu}(\alpha, h)$ and by Theorem 2.1, we have

$$
\Psi(z)=-\frac{z\left(g_{n}^{\mu}(\alpha)(z)\right)^{\prime}}{p g_{n}^{\mu}(\alpha)(z)} \prec h(z),
$$

and letting

$$
q(z)=-\frac{z\left(H_{p, \beta, \mu}^{\alpha} f(z)\right)^{\prime}}{p g_{n}^{\mu}(\alpha)(z)},
$$

we can complete the proof as in Theorem 2.4. Next, let

$$
F_{p, \delta}(f(z))=\frac{\delta-p}{z^{\delta}} \int_{0}^{z} t^{\delta-1} f(t) d t \quad(\delta>0),
$$

which by using (1.6) gives

$$
\left.\delta H_{p, \beta, \mu}^{\alpha} F_{p, \delta} f(z)+z\left(H_{p, \beta, \mu+1}^{\alpha}\right) F_{p, \delta} f(z)\right)^{\prime}=(\delta-p) H_{p, \beta, \mu}^{\alpha} f(z) .
$$

The operator $F_{p, \delta}$ was investigated by many authors (see $[10,11]$ ).

Theorem 2.6. Let $h \in \mathbb{P}$ with $\Re\{\delta-p h(z)\}>0$ and $f \in S_{n}^{\mu}(\alpha, h)$, then $F_{p, \delta}(f) \in$ $S_{n}^{\mu}(\alpha, h)$ provided $F_{n}^{\mu}(\alpha) \neq 0$, where $F_{n}^{\mu}(\alpha)$ is defined as in (1.10).

Proof. From (2.29), we have

$$
\delta F_{n}^{\mu}(\alpha)(z)+z\left(F_{n}^{\mu}(\alpha)(z)\right)^{\prime}=(\delta-p) f_{n}^{\mu}(\alpha)(z) .
$$

Let

$$
q(z)=-\frac{z\left(H_{p, \beta, \mu}^{\alpha} F_{p, \delta}(f(z))\right)^{\prime}}{p F_{n}^{\mu}(\alpha)(z)}
$$


and

$$
w(z)=-\frac{z\left(F_{n}^{\mu}(\alpha)(z)\right)^{\prime}}{p F_{n}^{\mu}(\alpha)(z)} .
$$

Using (2.30) in (2.31), we have

$$
\delta-p w(z)=(\delta-p) \frac{f_{n}^{\mu}(\alpha)(z)}{F_{n}^{\mu}(\alpha)(z)} .
$$

Differentiating and using Theorem 2.1, we obtain

$$
w(z)+\frac{z w^{\prime}(z)}{\delta-p w(z)}=-\frac{z\left(f_{n}^{\mu}(\alpha)(z)\right)^{\prime}}{p f_{n}^{\mu}(\alpha)(z)} \prec h(z) .
$$

By Lemma 1.2, (2.32) implies $w(z) \prec h(z)$. The remaining part of the proof is similar to that of Theorem 2.2, so we omit it.

The proof of the following theorem is similar to that of Theorems 2.3 and 2.5, so we omit it.

Theorem 2.7. Let $h \in \mathbb{P}$ with $\Re\{\delta-p h(z)\}>0$ and $f \in K_{n}^{\mu}(\alpha, h)$, with respect to $g_{n}^{\mu} \in S_{n}^{\mu}(\alpha, h)$, then, $F_{p, \delta}(f) \in K_{n}^{\mu}(\alpha, h)$ with respect to $G=F_{p, \delta}(g)$ provided $G_{n}^{\mu}(\alpha)(z) \neq 0$.

Note that for $h(z)=\frac{1+A z}{1+B z},-1 \leq B<A \leq 1$, we have $\Re h(z)=\frac{1+A}{1+B}$.

Remark 2.8. Taking $h(z)=\frac{1+A z}{1+B z}$, in Theorems 2.2-2.7 we get corresponding results for the classes $S_{n}^{\mu}(\alpha, A, B)$ and $K_{n}^{\mu}(\alpha, A, B)$.

Theorem 2.9. If $h \in \mathbb{P}$, with $\Re\{p+1-\gamma-p h(z)\}>0, f \in S_{n}^{\mu}(\alpha, h), \varphi \in \sum_{p}$ and $z^{p+1} \varphi(z) \in R(\gamma), \gamma<1$, then $f * \varphi \in S_{n}^{\mu}(\alpha, h)$.

Proof. For $f \in S_{n}^{\mu}(\alpha, h)$, we have

$$
F(z)=-\frac{z\left(H_{p, \beta, \mu}^{\alpha} f(z)\right)^{\prime}}{p f_{n}^{\mu}(\alpha)(z)} \prec h(z) .
$$

Let

then $\psi \in \mathbb{A}$ and

$$
\psi(z)=z^{p+1} f_{n}^{\mu}(\alpha)(z),
$$

$$
\frac{z \psi^{\prime}(z)}{\psi(z)}=p+1+\frac{z\left(f_{n}^{\mu}(\alpha)(z)\right)^{\prime}}{f_{n}^{\mu}(\alpha)(z)} \prec p+1-p h(z) .
$$

From the hypotheses of the theorem, we see that

$$
\Re \frac{z \psi^{\prime}(z)}{\psi(z)}>\gamma,
$$

that is $\psi \in S^{*}(\gamma), \gamma<1$. For $\varphi \in \sum_{p}$ it is easy to get

$$
z^{p+1} H_{p, \beta, \mu}^{\alpha}(f * \varphi)\left(\varepsilon_{k}^{j} z\right)=\left(z^{p+1} \varphi(z)\right) * H_{p, \beta, \mu}^{\alpha} f\left(\varepsilon_{k}^{j} z\right)
$$

and

$$
z^{p+2}\left(H_{p, \beta, \mu}^{\alpha}(f * \varphi)(z)\right)^{\prime}=\left(z^{p+1} \varphi(z)\right) *\left(z^{p+2} H_{p, \beta, \mu}^{\alpha} f(z)\right)^{\prime}
$$


So, we have

$$
\begin{aligned}
\Psi(z) & =-\frac{\left(H_{p, \beta, \mu}^{\alpha}(f * \varphi)(z)\right)^{\prime}}{\frac{p}{k} \sum_{j=0}^{k-1} \varepsilon_{k}^{j p} H_{p, \beta, \mu}^{\alpha}(f * \varphi)\left(\varepsilon_{k}^{j} z\right)} \\
& =-\frac{\left(z^{p+1} \varphi(z)\right) * z^{p+2}\left(H_{p, \beta, \mu}^{\alpha} f(z)\right)^{\prime}}{p z^{p+1} \varphi(z) *\left(z^{p+1} f_{n}^{\mu}(\alpha)(z)\right)} \\
& =\frac{z^{p+1} \varphi(z) *(\psi(z) F(z))}{z^{p+1} \varphi(z) * \psi(z)} .
\end{aligned}
$$

Since $h$ is convex, univalent, applying Lemma 1.4, it follows $\Psi(z) \prec h(z)$, that is $f * \varphi \in S_{n}^{\mu}(\alpha, h)$.

Remark 2.10. Taking $\mu=1$, in the above results we obtain results concerning the operator $H_{p, \beta}^{\alpha} f(z)$ defined by (1.9).

Acknowledgement. The authors thank the referees for their valuable suggestions which led to the improvement of the paper.

\section{References}

[1] Aqlan, E., Jahangiri, J.M., Kulkarni, S.R., Certain integral operators applied to meromorphic p-valent functions, J. Nat. Geom., 24(2003), 111-120.

[2] Bulboaca, T., Differential Subordinations and Superordinations, Recent Results, House of Scientific Book Publ., Cluj-Napoca, 2005.

[3] Eenigenberg, P., Miller, S.S., Mocanu, P.T., Reade, M.O., On Briot-Bouquet differential subordination, Gen. Inequal., 3(1983), 339-348.

[4] Kumar, V., Shukla, S.L., Certain integrals for classes of p-valent meromorphic functions, Bull. Aust. Math. Soc., 25(1982), 85-97.

[5] Miller, S.S., Mocanu, P.T., Differential Subordination: Theory and Applications, Series on Monographs and Textbooks in Pure and Applied Mathematics, Vol. 225, Marcel Dekker Inc., New York and Basel, 2000.

[6] Miller, S.S., Mocanu, P.T., Differential subordinations and univalent functions, Michigan Math. J., 28(1981), no. 2, 157-171.

[7] Miller, S.S., Mocanu, P.T., Differential subordinations and inequalities in the complex plane, J. Differential Equations, 67(1987), 199-211.

[8] Mostafa, A.O., Inclusion results for certain subcasses of p-valent meromorphic functions associated with a new operator, J. Ineq. Appl., 169(2012), 1-14.

[9] Ruscheweyh, S., Convolutions in Geometric Function Theory, Séminaire de Mathématiques Supérieures, vol. 83, Les Presses de l'Université de Montréal, Montreal, Quebec, 1982.

[10] Uralegaddi, B.A., Somanatha, C., Certain classes of meromorphic multivalent functions, Tamkang J. Math., 23(1992), 223-231.

[11] Yang, D.G., Certain convolution operators for meromorphic functions, South. Asian Bull. Math., 25(2001), 175-186. 
Adela O. Mostafa

Department of Mathematics, Faculty of Science

Mansoura University, Mansoura 35516, Egypt

e-mail: adelaeg254@yahoo.com

Mohamed K. Aouf

Department of Mathematics, Faculty of Science

Mansoura University, Mansoura 35516, Egypt

e-mail: mkaouf 127@yahoo.com 